\title{
248.
}

\section{SKETCH OF A PROOF OF THE THEOREM THAT EVERY ALGEBRAIC EQUATION HAS A ROOT.}

[From the Philosophical Magazine, vol. xviII. (1859), pp. 436-439.]

I HAVE referred to the theorem as usually stated; for it is an easy consequence of the existence of a single root of an equation of any order, that for an equation of the $n$th order there are $n$ roots: the proof here proposed goes, however, to show directly the existence of the $n$ roots: it is in form a geometrical one, and was suggested to me some months ago by a letter from Prof. De Morgan, containing the remark made in his memoir, "A Proof of the Existence of a Root in every Algebraic Equation," \&c. (Camb. Phil. Trans. vol. x. 1858), viz. "that the curves $P=0, Q=0$, the intersections whereof determine the root-points, are such that two branches, one of each curve, cannot enclose a space." The proof which occurred to me was in character somewhat similar to that given by the Astronomer Royal in the paper, "Suggestion of a Proof of the Theorem that every Algebraic Equation has a Root" (Camb. Phil. Trans. vol. x. 1858), and which was suggested to him by Prof. De Morgan's memoir. I have since varied my proof by considering therein cones in the place of plane curves. It will be obvious, upon reading it, that the proof is closely connected with Cauchy's well-known theorem for the number of roots within a given circuit; the circuit being in this case infinity, and the number of roots included within it consequently equal to the order of the equation.

The curve represented by an equation of the $n$th degree between the coordinates $(x, y)$ is by definition a curve of the $n$th order; and a cone standing on any such curve (taking the vertex for origin) is represented by a homogeneous equation of the $n$th degree between the coordinates $(x, y, z)$, and is by definition a cone of the $n$th order. It is very easy to show that an equation of the $n$th degree cannot have more than $n$ roots; and we have thence the geometrical theorems, that a curve of the $n$th 
order is not intersected by a line in more than $n$ points, and that a cone of the $n$th order is not intersected by a plane (I speak throughout of planes through the vertex) in more than $n$ lines. I assume that an algebraic curve is always a continuous curve, viz. that it consists of a branch or branches, no one of which is a courbe pointillée, or a branch terminating abruptly in a point; an algebraic cone will be in the like sense a continuous surface. An algebraic curve cannot be an indefinite spiral, for in that case there would be lines meeting it in an infinity of points; and in like manner an algebraic cone cannot be an indefinite spiral surface: an algebraic cone consists, therefore, of a closed sheet or sheets. An algebraic curve may indeed have conjugate or isolated points, and an algebraic cone have conjugate or isolated lines: this is a circumstance which will be adverted to in the sequel. It will fix the ideas as to the general form of an algebraic cone, to remark that it may comprise twin-pair sheets, such as the sheet of a cone of the second order (this is properly spoken of as a twin-pair sheet, each of the two opposite portions of it being called, for distinction, a twin-sheet); and of single sheets, such as one at least of the sheets of a cone of the third or any other odd order (see the annexed "Note upon Cones of the Third Order," [249]). The advantage of the consideration of cones instead of plane curves, is that we have only closed sheets, and thus get rid of the distinction which exists for plane curves between infinite branches and the branches which are closed curves.

My proof depends on the following lemma, viz. "Consider two algebraic cones with the same vertex, each of them of the order $n$; then if there be some one plane meeting the first cone in $n$ lines, and the second cone in $n$ lines, such that the lines of each set occur alternately, the two cones intersect in at least $n$ lines."

The truth of this lemma is, I conceive, a matter of intuition, depending only on the notion of the continuity of the sheets of the surface. Thus, if we have in plano, through a point $O$, the lines $A, A^{\prime}$ and $B, B^{\prime}$, such that, $A$, $\alpha$ being opposite points on the same line, and so for the other lines, the order round $O$ is $A, B, A^{\prime}, B^{\prime}$, $\alpha, \beta, \alpha^{\prime}, \beta^{\prime}$, it is obvious that we cannot through the lines $A, A^{\prime}$ draw a cone, and through the lines $B, B^{\prime}$ draw a cone, without making these cones intersect in at least two lines: and in like manner for two sets, each of $n$ lines. I have, in the enunciation of the lemma, said that the cones are each of them of the order $n$; this was necessary in order to exclude a case which might otherwise have happened, viz. a line of intersection of the plane with either of the cones might have been a conjugate or isolated line without any sheet through it; and if this were so, we could not infer the existence of the $n$ lines of intersection of the two cones. But if a plane meet an algebraic cone of the $n$th order in $n$ lines, no one of these can be a conjugate or isolated line; for such line is to be considered as two or more coincident lines, and there would be in all more than $n$ lines of intersection of the plane and cone.

Consider now the equation

$$
\phi u=0,
$$


where $\phi u$ is a rational and integral function of $u$ with (in general) imaginary coefficients, and write

$$
\phi \cdot(x+y \sqrt{-1})=P+Q \sqrt{-1},
$$

$P, Q$ being real functions of $(x, y)$, each of them of the degree $n$; if $(x, y)$ are rectangular coordinates, then $P=0, Q=0$ are real curves each of the order $n$. And to each point of intersection of the two curves there corresponds a root of the equation. The two curves do not intersect in more than $n$ points (for if they did, the equation $\phi u=0$ would have more than $n$ roots); hence if it be shown that the two curves intersect in at least $n$ points, they will intersect in precisely $n$ points, and the equation will have $n$ roots. Take any point as the common vertex of two cones standing upon the curves $P=0, Q=0$ respectively; each point of intersection of the two curves corresponds to a line of intersection of the two cones, and it is only necessary to show that the two cones intersect in at least $n$ lines Take for the vertex a point in the perpendicular at the origin of $(x, y)$ to the plane of the two curves, and at a distance unity from such origin, viz. a point such that, treating it as the origin of the coordinates $(x, y, z)$, the coordinates in respect thereto of the origin $(x, y)$ are $x=0, y=0, z=1$. The equations of the cones are at once deduced from those of the curves by writing therein $\left(\frac{x}{z}, \frac{y}{z}\right)$ in the place of $(x, y)$ and, to render the equation integral, multiplying by $z^{n}$; or if $P^{\prime}=0, Q^{\prime}=0$ are the equations of the cones, we have

$$
z^{n} \phi\left(\frac{x+y \sqrt{-1}}{z}\right)=P^{\prime}+Q^{\prime} \sqrt{-1}
$$

Consider the section by the plane through the vertex parallel to the plane of the two curves: the equation of this plane is $z=0$; and it is clear that, to obtain the intersections of this cone with the plane in question, we have only in $\phi\left(\frac{x+y \sqrt{-1}}{z}\right)$ to disregard all the terms after the first. Suppose that

$$
\phi u=(a+b \sqrt{-1}) u^{n}+\& c .
$$

then putting

$$
(a+b \sqrt{-1})(x+y \sqrt{-1})^{n}=P_{0}^{\prime}+Q_{0}^{\prime} \sqrt{-1},
$$

the equations $P_{0}^{\prime}=0, Q_{0}^{\prime}=0$ determine the intersections of the plane $z=0$ with the cones $P=0, Q=0$ respectively. But writing

$$
\begin{aligned}
& a+b \sqrt{-1}=A(\cos \alpha+\sqrt{-1} \sin \alpha), \\
& x+y \sqrt{-1}=r(\cos \theta+\sqrt{-1} \sin \theta),
\end{aligned}
$$

we have

$$
A r^{n}\{\cos (n \theta+\alpha)+\sqrt{-1} \sin (n \theta+\alpha)\}=P_{0}^{\prime}+Q_{0}^{\prime} \sqrt{-1}
$$


so that

$$
\text { - } P_{0}^{\prime}=A r^{n} \cos (n \theta+\alpha), \quad Q_{0}^{\prime}=A r^{n} \sin (n \theta+\alpha),
$$

or the intersections with the cone $P=0$ are the $n$ lines given in direction by the equation

$$
n \theta+\alpha=\left(m+\frac{1}{2}\right) \pi,
$$

and the intersections with the cone $Q=0$ are the $n$ lines given in direction by the equation

$$
n \theta+\alpha=m \pi
$$

in each of which equations $m$ is any integer number from 0 to $n-1$. Hence the plane $z=0$ meets the cones in two sets of lines succeeding each other alternately, as required by the lemma, and the two cones intersect in at least $n$ lines. And it is thus shown that the given equation of the $n$th degree has $n$ roots.

2, Stone Buildings, W.C., September 26, 1859. 